Florian A. Potra

\title{
Q-superlinear convergence of the iterates in primal-dual interior-point methods ${ }^{\star}$
}

Received: February 9, 2000 / Accepted: February 20, 2001

Published online May 3, 2001 - (C) Springer-Verlag 2001

\begin{abstract}
Sufficient conditions are given for the Q-superlinear convergence of the iterates produced by primal-dual interior-point methods for linear complementarity problems. It is shown that those conditions are satisfied by several well known interior-point methods. In particular it is shown that the iteration sequences produced by the simplified predictor-corrector method of Gonzaga and Tapia, the simplified largest step method of Gonzaga and Bonnans, the LPF+ algorithm of Wright, the higher order methods of Wright and Zhang, Potra and Sheng, and Stoer, Wechs and Mizuno are Q-superlinearly convergent.
\end{abstract}

Key words. linear complementarity problem - interior-point algorithm - sufficient matrices superlinear convergence

\section{Introduction}

Most of the work on primal-dual interior-point methods has been concerned with the convergence to zero of the duality gap as illustrated by the recent monographs [20], [27], [29]. Comparatively there are few results on the convergence of the iteration sequences produced by such algorithms to the solution of the problem. A first result in this direction was obtained by Tapia, Zhang and Ye [24] who gave sufficient conditions for the convergence of the iteration sequence generated by the Kojima-Mizuno-Yoshise [11] primaldual interior-point algorithm. However they did not prove that those sufficient conditions can be satisfied by a specific realization of the Kojima-Mizuno-Yoshise algorithm in such a way that the polynomial complexity of the algorithm is preserved. Gonzaga and Tapia [9] showed that the iterates produced by Mizuno-Todd-Ye predictor-corrector method [14] converge to the analytic center of the solution set. We note that the MizunoTodd-Ye predictor-corrector method (MTY) requires two matrix factorization and two backsolves per iteration and has $O(\sqrt{n} L)$ iteration complexity [14] and Q-quadratically convergent duality gap [30]. Gonzaga and Tapia [10] also proposed a modification of the MTY predictor-corrector method (called the simplified MTY method) that asymptotically requires only one matrix factorization per iteration while retaining the $O(\sqrt{n} L)$ iteration complexity and the Q-quadratic convergence of the duality gap, and showed that the iteration sequence produced by this method converges, but not necessarily to the analytic center. The above results about the convergence of the iteration sequence

F.A. Potra: Department of Mathematics and Statistics, University of Maryland Baltimore County, e-mail: potra@math.umbc.edu

Mathematics Subject Classification (1991): 49M15, 65K05, 90C33

^ This work was supported in part by NSF, Grant DMS-9996154 . 
were extended for a very general class of feasible interior-point methods by Bonnans and Gonzaga [2] and generalized for infeasible interior-point methods by Bonnans and Potra [4]. From the last two papers it follows that virtually all interior-point methods using an $l_{2}$ neighborhood of the central path produce convergent iteration sequences. It also follows that virtually all interior-point methods using $l_{\infty}$ neighborhoods of the central path and search directions that approach asymptotically the affine scaling direction produce convergent iteration sequences. Thus the problem of the convergence of the iterates was established for a large class of interior point methods. However none of the above mentioned papers establishes the rate of convergence of the iteration sequence. Among the very few results about the rate of convergence of the iteration sequence we mention the paper of Wright [26] where it is shown that the iteration sequence produced by the LPF+ algorithm is R-subquadratically convergent for nondegenerate monotone linear complementarity problems, and the paper of Potra and Sheng [18] that analyzes an interior point method for linear complementarity problems with sufficient matrices whose iteration sequence is Q-subquadratically convergent for nondegenerate problems and convergent with Q-order 1.25 otherwise. We note that for many interior point methods it is possible to prove R-superlinear convergence of the distance from the iterates to the solution set (see, e.g. [23]). Even if the sequence of iterates is convergent towards a solution, the fact that the distance between the iterates to the solution set converges R-superlinearly does not imply that the iterates converge R-superlinearly. This aspect will be addressed at the end of Sect. 4.

In the present paper we give sufficient conditions for the Q-superlinear convergence of the iterates produced by primal-dual interior-point methods for sufficient linear complementarity problems. We show that those conditions are satisfied by several well known interior point methods. In particular we show that if the problem has a strictly complementary solution then: the simplified predictor-corrector method of Gonzaga and Tapia [10] and the simplified largest step method of Gonzaga [8] produce a Qquadratically convergent iteration sequences; the LPF+ algorithm of Wright [26,27] produces a Q-subquadratically convergent iteration sequence; the methods of Wright and Zhang [28] and Potra and Sheng [17] that asymptotically require one matrix factorization and $p$ backsolves at each iteration produce iteration sequences that are convergent with $\mathrm{Q}$-order $p+1$. The high order simplified largest step method of Gonzaga and Bonnans [3] asymptotically requires one matrix factorization and $2 p$ backsolves per iteration. In [3] it is shown that for this method the duality gap converges to zero with strong Q-order at least $p+1$. From our analysis it follows that the iteration sequence is Q-superlinearly convergent with the same order. Recently Stoer, Wechs and Mizuno [22] proposed an interior-point method based on high order derivatives of the central path that requires asymptotically one matrix factorization and $p$ backsolves per iteration. Their algorithm depends on a constant $\zeta \in(0,1)$. It is shown that the primal-dual gap converges to zero with strong Q-order at least $p+1-\zeta$ and the iteration sequence converges with the same R-order. From our analysis it will follow that if $\zeta(p+1-\zeta)>1$ then the iteration sequence converges with strong Q-order $\zeta(p+1-\zeta)$.

For problems that do not have a strictly complementary solution we show that the iteration sequences produced by the method of Potra and Sheng [19] are convergent with Q-order 1.25 and those produced by the method of Stoer, Wechs and Mizuno are convergent with strong Q-order at least $\zeta(p+1-\zeta) / 2$. 
We would like to stress that establishing Q-superlinear convergence of the iterates may have an important impact on designing alternative stopping criteria for interior-point methods. Presently most interior-point methods terminate when an iterate $z^{k}=\left(s^{k}, x^{k}\right)$ with duality gap less than a given tolerance $\epsilon>0$ is produced. Under certain conditions this implies that the distance between $z^{k}$ and the solution set is $O(\epsilon)$, but the constant hidden in the $O(\cdot)$ notation cannot be estimated in practice. On the other hand if $z^{k}$ converges Q-superlinearly to a solution $z^{*}$, then $\left\|z^{k}-z^{*}\right\|$ has asymptotically the same value as $\left\|z^{k}-z^{k+1}\right\|$ (in the sense that $\lim \left(\left\|z^{k}-z^{*}\right\| /\left\|z^{k}-z^{k+1}\right\|\right)=1$ ), so that efficient stopping criteria can be written to ensure that the interior point method terminates with a $z^{k}$ at distance less than $\epsilon$ from the solution.

The paper is organized as follows. In Sect. 2 we review some facts about Rconvergence and Q-convergence. The main results of the paper are contained in Sect. 3 where sufficient conditions for the superlinear convergence of the iteration sequences produced by primal-dual methods for $P_{*}$ linear complementarity problems are given in a very general framework. Section 4 contains applications to some particular primal-dual interior-point methods.

Conventions. We denote by $\mathbb{N}$ the set of all nonnegative integers. $\mathbb{R}, \mathbb{R} \boldsymbol{R}_{+}, \mathbb{R} \boldsymbol{R}_{++}$denote the set of real, nonnegative real, and positive real numbers respectively. The natural $\log$ arithm of a positive real number $\alpha$ is denoted by $\log \alpha$. Given a vector $x$, the corresponding upper case symbol denotes as usual the diagonal matrix $X$ defined by the vector. The symbol $e$ represents the vector of all ones, with dimension given by the context.

We denote component-wise operations on vectors by the usual notations for real numbers. Thus, given two vectors $u, v$ of the same dimension, $u v, u / v$, etc. will denote the vectors with components $u_{i} v_{i}, u_{i} / v_{i}$, etc. This notation is consistent as long as component-wise operations always have precedence in relation to matrix operations. Note that $u v \equiv U v$ and if $A$ is a matrix, then $A u v \equiv A U v$, but in general $A u v \neq(A u) v$. We denote the nullspace and range space of a matrix $A$ by $\mathcal{N}(A)$ and $\mathcal{R}(A)$ respectively.

We frequently use the $O(\cdot)$ and $\Omega(\cdot)$ notation to express the relationship between functions. Our most common usage will be associated with a sequence $\left\{x^{k}\right\}$ of vectors and a sequence $\left\{\tau_{k}\right\}$ of positive real numbers. In this case $x^{k}=O\left(\tau_{k}\right)$ means that there is a constant $K$ (dependent on problem data) such that for every $k \in \mathbb{N},\left\|x^{k}\right\| \leq K \tau_{k}$. Similarly, if $x^{k}>0, x^{k}=\Omega\left(\tau_{k}\right)$ means that $\left(x^{k}\right)^{-1}=O\left(1 / \tau_{k}\right)$. Finally, $x^{k}=\Theta\left(\tau_{k}\right)$ means that $x^{k}=O\left(\tau_{k}\right)$ and $x^{k}=\Omega\left(\tau_{k}\right)$.

We use the same notations for a point $x$ in a set parameterized by $\tau$, say $\mathcal{E}_{\tau}$. We say that $x=O(\tau)$ (resp. $x=\Omega(\tau), x=\Theta(\tau)$ ) whenever there is a constant $K$ such that $\|x\| \leq K \tau$ (resp. $\left.x^{-1}=O(1 / \tau), x=\Theta(\tau)\right)$ for all $x \in \mathcal{E}_{\tau}$, and all small enough $\tau$. In particular, $x=\Theta(1)$ in $\mathcal{E}_{\tau}$ means that there are constants $K_{2}>K_{1}>0$, such that any $x \in \mathcal{E}_{\tau}$ satisfies $K_{1} \leq x_{i} \leq K_{2}, i=1, \ldots, n$.

Given a vector $x \in \mathbb{R}^{n}$ and an index set $P \subset\{1,2, \ldots, n\}$ we denote by $x_{P}$ the vector with components $x_{i}, i \in P$. 


\section{R-order and Q-order of convergence}

In this section we review some notions related to the R-order and Q-order of convergence. For more details see [16]. First we give the definition of R-linear and Q-linear convergence.

Definition 1. Let $\left(\alpha_{k}\right)$ be a sequence of positive real numbers. We say that:

1. $\left(\alpha_{k}\right)$ converges $R$-linearly if

$$
\lim \sup \sqrt[k]{\alpha_{k}}<1
$$

2. $\left(\alpha_{k}\right)$ converges $R$-linearly with global rate $\rho<1$ if

$$
\alpha_{k}=O\left(\rho^{k}\right) .
$$

Definition 2. Let $\left(\alpha_{k}\right)$ be a sequence of positive real numbers. We say that:

1. $\left(\alpha_{k}\right)$ converges $Q$-linearly if

$$
\lim \sup \frac{\alpha_{k+1}}{\alpha_{k}}<1
$$

2. $\left(\alpha_{k}\right)$ converges $Q$-linearly with global rate $\rho<1$ if

$$
\alpha_{k+1}<\rho \alpha_{k} \forall k \in \mathbb{N} \text {. }
$$

First, we observe that if the sequence $\left(\alpha_{k}\right)$ satisfies any of the conditions above then $\left(\alpha_{k}\right)$ converges to zero. Second, we note that if $\left(\alpha_{k}\right)$ converges Q-linearly then it also converges R-linearly since

$$
\lim \sup \sqrt[k]{\alpha_{k}} \leq \limsup \frac{\alpha_{k+1}}{\alpha_{k}} .
$$

Moreover if $\left(\alpha_{k}\right)$ converges Q-linearly with global rate $\rho$ then it also converges R-linearly with global rate $\rho$. We note the following difference in between R-linear convergence and Q-linear convergence: while R-linear convergence implies global R-linear convergence with some global rate $\rho<1$ (we can take any $\rho \in\left(\lim \sup \sqrt[k]{\alpha_{k}}, 1\right)$ ), the corresponding property does not hold for Q-linear convergence.

Definition 3. Let $\left(\alpha_{k}\right)$ be a sequence of positive real numbers. We say that $\left(\alpha_{k}\right)$ converges $R$-superlinearly if

$$
\lim \sqrt[k]{\alpha_{k}}=0
$$

Definition 4. Let $\left(\alpha_{k}\right)$ be a sequence of positive real numbers. We say that $\left(\alpha_{k}\right)$ converges $Q$-superlinearly if

$$
\lim \frac{\alpha_{k+1}}{\alpha_{k}}=0
$$

Obviously R-superlinear convergence implies R-linear convergence, and Q-superlinear convergence implies Q-linear convergence. Also Q-superlinear convergence implies R-superlinear convergence. 
Definition 5. Let $\left(\alpha_{k}\right)$ be a sequence of positive real numbers. The R-order of convergence of $\left(\alpha_{k}\right), \mathcal{R}\left(\left(\alpha_{k}\right)\right)$, is defined by

$$
\mathcal{R}\left(\left(\alpha_{k}\right)\right):=\liminf \sqrt[k]{\left|\log \left(\alpha_{k}\right)\right|} ;
$$

Definition 6. Let $\left(\alpha_{k}\right)$ be a sequence of positive real numbers. The Q-order of convergence of $\left(\alpha_{k}\right), \mathcal{Q}\left(\left(\alpha_{k}\right)\right)$, is defined by

$$
\mathcal{Q}\left(\left(\alpha_{k}\right)\right):=\liminf \frac{\left|\log \alpha_{k+1}\right|}{\left|\log \alpha_{k}\right|} .
$$

It is easily seen that for any sequence of positive real numbers we have

$$
\mathcal{Q}\left(\left(\alpha_{k}\right)\right) \leq \mathcal{R}\left(\left(\alpha_{k}\right)\right)
$$

In Definitions 5 and 6 we have not assumed that the sequence $\left(\alpha_{k}\right)$ converges to zero. From the following proposition it follows that if $\left(\alpha_{k}\right)$ is bounded and $\mathcal{R}\left(\left(\alpha_{k}\right)\right)>1$ then $\left(\alpha_{k}\right)$ converges to zero.

Proposition 1. Assume that the sequence $\left(\alpha_{k}\right)$ is bounded. Then

1. If $\mathcal{R}\left(\left(\alpha_{k}\right)\right)>1$ then $\left(\alpha_{k}\right)$ is $R$-superlinearly convergent.

2. If $\mathcal{Q}\left(\left(\alpha_{k}\right)\right)>1$ then $\left(\alpha_{k}\right)$ is $Q$-superlinearly convergent.

Proof. 1) From the hypothesis it follows that for any $\omega \in\left(1, \mathcal{R}\left(\left(\alpha_{k}\right)\right)\right)$ there is $K \in \mathbb{N}$ such that

$$
\left|\log \left(\alpha_{k}\right)\right|>\omega^{k}, \forall k \geq K
$$

Therefore $\lim \left|\log \left(\alpha_{k}\right)\right|=\infty$. Since $\left(\alpha_{k}\right)$ is bounded it follows that $\lim \log \left(\alpha_{k}\right)=-\infty$. Hence $\lim \alpha_{k}=0$. By taking $K$ large enough we have

$$
\alpha_{k}<1, \log \left(\alpha_{k}\right)<-\omega^{k}, \forall k \geq K \text {. }
$$

Consequently

$$
\alpha_{k}<\exp \left(-\omega^{k}\right), \forall k \geq K,
$$

and this shows that $\lim \sqrt[k]{\alpha_{k}}=0$.

2) From (1) and the proof above we deduce that $\lim \alpha_{k}=0$. It follows that for any $\omega \in\left(1, \mathcal{Q}\left(\left(\alpha_{k}\right)\right)\right)$ there is $K \in \mathbb{N}$ such that

$$
\alpha_{k}<1, \log \left(\alpha_{k+1}\right)<\omega \log \left(\alpha_{k}\right), \forall k \geq K \text {. }
$$

It follows that

$$
\frac{\alpha_{k+1}}{\alpha_{k}}<\alpha_{k}^{\omega-1}
$$

Since $\lim \alpha_{k}=0$ this shows that $\left(\alpha_{k}\right)$ is Q-superlinearly convergent. 
By examining the proof of the above proposition we can easily prove the following result.

Proposition 2. Let $\left(\alpha_{k}\right)$ be a bounded sequence of positive real numbers.

1. If $\mathcal{R}\left(\left(\alpha_{k}\right)\right)>1$ then for any $\omega \in\left(1, \mathcal{R}\left(\left(\alpha_{k}\right)\right)\right)$ there is a constant $r_{\omega}$ such that

$$
\alpha_{k} \leq r_{\omega} \exp \left(-\omega^{k}\right), \forall k \in \mathbb{N}
$$

2. If $\mathcal{Q}\left(\left(\alpha_{k}\right)\right)>1$ then for any $\omega \in\left(1, \mathcal{Q}\left(\left(\alpha_{k}\right)\right)\right)$ there is a constant $q_{\omega}$ such that

$$
\alpha_{k+1} \leq q_{\omega} \alpha_{k}^{\omega}, \forall k \in \mathbb{N} .
$$

The above proposition motivates the following definitions.

Definition 7. Let $\left(\alpha_{k}\right)$ be a sequence of positive real numbers that converges to zero and let $\omega>1$ be a constant. We say that $\left(\alpha_{k}\right)$ converges with strong $R$-order at least $\omega$ if there is a constant $0<\gamma<1$ such that

$$
\alpha_{k}=O\left(\gamma^{\omega^{k}}\right)
$$

Definition 8. Let $\left(\alpha_{k}\right)$ be a sequence of positive real numbers that converges to zero and let $\omega>1$ be a constant. We say that $\left(\alpha_{k}\right)$ converges with strong $Q$-order at least $\omega$ if

$$
\alpha_{k+1}=O\left(\alpha_{k}^{\omega}\right)
$$

Using Proposition 2 we can easily prove the following result.

Proposition 3. Assume that the sequence $\left(\alpha_{k}\right)$ is bounded.

1. If $\mathcal{R}\left(\left(\alpha_{k}\right)\right)>1$ then $\left(\alpha_{k}\right)$ converges to zero with strong $R$-order at least $\omega$ for any $\omega \in\left(1, \mathcal{R}\left(\left(\alpha_{k}\right)\right)\right.$, and

$$
\mathcal{R}\left(\left(\alpha_{k}\right)\right)=\sup \left\{\omega>1 \mid\left(\alpha_{k}\right) \text { converges with strong } R \text {-order at least } \omega\right\} .
$$

2. If $\mathcal{Q}\left(\left(\alpha_{k}\right)\right)>1$ then $\left(\alpha_{k}\right)$ converges to zero with strong $Q$-order at least $\omega$ for any $\omega \in\left(1, \mathcal{Q}\left(\left(\alpha_{k}\right)\right)\right.$, and

$$
\mathcal{Q}\left(\left(\alpha_{k}\right)\right)=\sup \left\{\omega>1 \mid\left(\alpha_{k}\right) \text { converges with strong } Q \text {-order at least } \omega\right\} \text {. }
$$

A special situation arises when the supremum in (4) and/or in (5) is attained.

Definition 9. Let $\left(\alpha_{k}\right)$ be a sequence of positive real numbers that converges to zero and let $\omega>1$ be a constant. We say that $\mathcal{R}\left(\left(\alpha_{k}\right)\right)$ is the exact $R$-order of $\left(\alpha_{k}\right)$, if $\mathcal{R}\left(\left(\alpha_{k}\right)\right)>1$ and $\left(\alpha_{k}\right)$ converges with strong $R$-order at least $\mathcal{R}\left(\left(\alpha_{k}\right)\right)$.

Definition 10. Let $\left(\alpha_{k}\right)$ be a sequence of positive real numbers that converges to zero and let $\omega>1$ be a constant. We say that $\mathcal{Q}\left(\left(\alpha_{k}\right)\right)$ is the exact $Q$-order of $\left(\alpha_{k}\right)$, if $\mathcal{Q}\left(\left(\alpha_{k}\right)\right)>1$ and $\left(\alpha_{k}\right)$ converges with strong $Q$-order at least $\mathcal{Q}\left(\left(\alpha_{k}\right)\right)$. 
Let us note that the notions of exact R-order and exact Q-order defined above are slightly more general than the corresponding notions defined in [16].

A particular but very important case is represented by the notions of subquadratic and quadratic convergence.

Definition 11. Let $\left(\alpha_{k}\right)$ be a sequence of positive real numbers that converges to zero.

1. We say that $\left(\alpha_{k}\right)$ converges $R$-subquadratically if $\mathcal{R}\left(\left(\alpha_{k}\right)\right) \geq 2$;

2. We say that $\left(\alpha_{k}\right)$ converges $R$-quadratically if $\left(\alpha_{k}\right)$ converges with strong $R$-order at least 2.

Definition 12. Let $\left(\alpha_{k}\right)$ be a sequence of positive real numbers that converges to zero.

1. We say that $\left(\alpha_{k}\right)$ converges $Q$-subquadratically if $\mathcal{Q}\left(\left(\alpha_{k}\right)\right) \geq 2$;

2. We say that $\left(\alpha_{k}\right)$ converges $Q$-quadratically if $\left(\alpha_{k}\right)$ converges with strong $Q$-order at least 2 .

In this paper we will be concerned with convergence properties of sequences of vectors. Let $\left(y^{k}\right)$ be a sequence of vectors belonging to a finite dimensional vector space $\mathbb{R}^{p}$ which converges to a vector $y^{*} \in \mathbb{R}^{p}$. Let $\|\cdot\|$ be any norm on $\mathbb{R}^{p}$. The convergence properties of the sequence of $\left(y^{k}\right)$ are by definition identical with the convergence properties of the sequence $\left(\left\|y^{k}-y^{*}\right\|\right)$. Thus we say that $\left(y^{k}\right)$ converges Q-quadratically if there is a constant $q_{2}$ such that

$$
\left\|y^{k+1}-y^{*}\right\| \leq q_{2}\left\|y^{k}-y^{*}\right\|^{2}, \forall k \in \mathbb{N} .
$$

We note that since any two norms on $\mathbb{R}^{p}$ are equivalent the (superlinear) convergence properties of a vector sequence are independent of the choice of the norm. This fact is an immediate consequence of the following result.

Proposition 4. Let $\left(\alpha_{k}\right)$ be a sequence of positive real numbers that converges to zero and let $\left(\beta_{k}\right)$ be another sequence of positive real numbers. Assume that there are three positive constants $\sigma, \underline{\beta}, \bar{\beta}$ and a positive integer $K$ such that

$$
\underline{\beta} \alpha_{k}^{\sigma} \leq \beta_{k} \leq \bar{\beta} \alpha_{k}^{\sigma}, \forall k \geq K .
$$

If $\left(\alpha_{k}\right)$ has any of the properties in Definitions $4,8,10$, and 12 then $\left(\beta_{k}\right)$ has the same property. In particular the $Q$-orders of convergence of $\left(\alpha_{k}\right)$ and $\left(\beta_{k}\right)$ are the same.

Proof. We only prove the fact that if $\left(\alpha_{k}\right)$ converges with strong Q-order at least $\omega$ so does $\left(\beta_{k}\right)$. From (3) and (7) it follows that there is a constant $q$ such that for sufficiently large $k$ we have

$$
\beta_{k+1} \leq \bar{\beta} \alpha_{k+1}^{\sigma} \leq \bar{\beta} q^{\sigma} \alpha_{k}^{\sigma \omega} \leq \frac{\bar{\beta} q^{\sigma}}{\underline{\beta}^{\omega}} \beta_{k}^{\omega},
$$

which proves our statement.

In the next proposition we give sufficient conditions under which $Q\left(\left(\alpha_{k}\right)\right)>1$ implies $Q\left(\left(\beta_{k}\right)\right)>1$, but the two Q-orders are no longer equal. 
Proposition 5. Let $\left(\alpha_{k}\right)$ be a sequence of positive real numbers that converges to zero and let $\left(\beta_{k}\right)$ be another sequence of positive real numbers. Assume that there are four positive constants $\sigma, \zeta, \underline{\beta}, \bar{\beta}$, with $0<\zeta<1$, and a positive integer $K$ such that

$$
\underline{\beta} \alpha_{k}^{\sigma} \leq \beta_{k} \leq \bar{\beta} \alpha_{k}^{\sigma \zeta}, \forall k \geq K
$$

If $\left(\alpha_{k}\right)$ converges with strong $Q$-order at least $\omega$ and if $\zeta \omega>1$ then $\left(\beta_{k}\right)$ converges with strong $Q$-order at least $\zeta \omega$. Also if $\zeta \mathcal{Q}\left(\left(\alpha_{k}\right)\right)>1$, then $\mathcal{Q}\left(\left(\beta_{k}\right)\right) \geq \zeta \mathcal{Q}\left(\left(\alpha_{k}\right)\right)$.

Proof. We prove only the fact that if $\left(\alpha_{k}\right)$ converges with strong Q-order at least $\omega$ then $\left(\beta_{k}\right)$ converges with strong Q-order at least $\zeta \omega$. From (3) and (8) it follows that there is a constant $q$ such that

$$
\beta_{k+1} \leq \bar{\beta} \alpha_{k+1}^{\sigma \zeta} \leq \bar{\beta} q^{\sigma \zeta} \alpha_{k}^{\sigma \zeta \omega} \leq \frac{\bar{\beta} q^{\sigma \zeta}}{\underline{\beta}^{\zeta \omega}} \beta_{k}^{\zeta \omega}
$$

for all sufficiently large $k$.

We have seen that if (7) holds and $\left(\alpha_{k}\right)$ is Q-superlinearly convergent, so is $\left(\beta_{k}\right)$. However if (8) holds and $\left(\alpha_{k}\right)$ is Q-superlinearly convergent then $\left(\beta_{k}\right)$ may not be Qsuperlinearly convergent. We also note that if (7) holds and $\left(\alpha_{k}\right)$ is Q-linearly convergent then $\left(\beta_{k}\right)$ may not be Q-linearly convergent. However we can easily prove the following result.

Proposition 6. Let $\left(\alpha_{k}\right)$ be a sequence of positive real numbers that converges to zero and let $\left(\beta_{k}\right)$ be another sequence of positive real numbers. Assume that there are two positive constants $\sigma$, and $\underline{\beta}$, and a positive integer $K$ such that

$$
0 \leq \beta_{k} \leq \bar{\beta} \alpha_{k}^{\sigma}, \forall k \geq K
$$

If $\left(\alpha_{k}\right)$ converges $R$-linearly so does $\left(\beta_{k}\right)$. Also if $\left(\alpha_{k}\right)$ converges $R$-linearly with global rate $\rho<1$ then $\left(\beta_{k}\right)$ converges $R$-linearly with global rate $\rho^{\sigma}$. Moreover if $\left(\alpha_{k}\right)$ has any of the properties defined in Definitions 3, 7, 9, and 11 then $\left(\beta_{k}\right)$ has the same property.

\section{Sufficient conditions for the superlinear convergence of the iterates}

In this paper we study the convergence of a class of infeasible interior point methods for solving the horizontal linear complementarity problem (HLCP):

$$
\begin{aligned}
x s & =0 \\
Q x+R s & =b \\
x, s & \geq 0,
\end{aligned}
$$

where $b \in \mathbb{R}^{n}$, and $Q, R \in \mathbb{R}^{n \times n}$ with $\operatorname{rank}[Q, R]=n$. 
We assume that there is a constant $\kappa \geq 0$ such that for any $u, v \in \mathbb{R}^{n}$,

$$
Q u+R v=0 \text { implies }(1+4 \kappa) \sum_{i \in \mathcal{I}_{+}(u, v)} u_{i} v_{i}+\sum_{i \in \mathcal{I}_{-}(u, v)} u_{i} v_{i} \geq 0
$$

where

$$
\mathcal{I}_{+}(u, v)=\left\{i: u_{i} v_{i}>0\right\}, \mathcal{I}_{-}(u, v)=\left\{i: u_{i} v_{i}<0\right\}
$$

or equivalently

$$
Q u+R v=0 \text { implies } u^{T} v \geq-4 \kappa \sum_{i \in \mathcal{I}_{+}(u, v)} u_{i} v_{i} .
$$

If the pair $(Q, R)$ satisfies (11) or (12) we say that $(Q, R)$ is a $P_{*}(\kappa)-$ pair. The class of all $P_{*}(\kappa)$-pairs is denoted by $P_{*}(\kappa)$, and the class $P_{*}$ is defined by

$$
P_{*}=\bigcup_{\kappa \geq 0} P_{*}(\kappa)
$$

i.e., $(Q, R)$ is a $P_{*}$-pair if $(Q, R) \in P_{*}(\kappa)$ for some $\kappa \geq 0$. It can be shown (see [25]) that the class $P_{*}$ defined above coincides with the class of sufficient pairs as defined in [5] (see also [21]).

If $(Q, R) \in P_{*}(0)$, we say that the linear complementarity problem (10) is monotone. It is known (see, e.g. [2]) that this problem trivially includes the linear programming problem (LP) and the convex quadratic programming problem (QP) in their usual formulations, and thus provides a quite general framework for the study of algorithms. Of course any LP and QP can also be written as a standard linear complementarity problem (SLCP) which is an HLCP where $R$ is the identity matrix and $-Q$ is positive semidefinite. For the equivalence between different formulations of $P_{*}(\kappa)$ linear complementarity problems see [1].

In [4] we described a general framework for analyzing the convergence of infeasible interior-point methods for monotone horizontal linear complementarity problems of the form (10) having a strictly complementary solution. In what follows we will consider some of the notions introduced there for the more general case where $(Q, R)$ is a $P_{*}$-pair and when (10) may not have a strictly complementary solution.

We define the measure of optimality of $(x, s) \in \mathbb{R}_{+}^{2 n}$ as

$$
\mu=\frac{1}{n} x^{T} s
$$

and its measure of feasibility as $\|r\|$ where $r$ is the residual in the linear part of (10),

$$
r=b-Q x-R s
$$

It is easily seen that the measure of optimality of $(x, s)$ is in fact the normalized $l_{1}$-norm of the residual of the nonlinear part of (10), $n \mu=\|x s\|_{1}$, so that finding a solution of HLCP (10) means finding a pair $(x, s) \in \mathbb{R}_{+}^{2 n}$ with $\mu=0$ and $r=0$. 
We consider algorithms for solving the HLCP (10) that follow approximately the "infeasible central path pinned on a" [4], defined as the set of triplets $(x, s, \tau)$ that satisfy

$$
\begin{aligned}
x s & =\tau e, \\
Q x+R s & =b-\tau a,
\end{aligned}
$$

where $a$ is a constant vector related to the starting point

$$
w^{0}=\left(x^{0}, s^{0}, \tau_{0}\right) \in \mathbb{R}_{++}^{2 n+1}
$$

by

$$
a=r^{0} / \tau_{0}
$$

with $r^{0}$ being the residual in the linear part of (10) at the starting point,

$$
r^{0}=b-Q x^{0}-R s^{0}
$$

From [21] it follows that if $(Q, R) \in P_{*}, \operatorname{rank}[Q, R]=n$, and (10) has a solution then (15) has a unique solution for any $0<\tau \leq \tau_{0}$.

It is easily seen that if $a$ is defined as above then the second equation of (15) is satisfied at the starting point. If we choose arbitrary $s^{0}>0, \tau_{0}>0$ and take $x^{0}=\tau_{0} / s^{0}$ then the first equation of (15) is also satisfied, i.e., the starting point chosen in this way belongs to the infeasible central path pinned on $a$. For any triplet $(x, s, \tau)$ belonging to the infeasible central path we have obviously $\mu=\tau$. Even if the starting point is on the infeasible central path, the subsequent points $(x, s, \tau)$ produced by the algorithm will not be on this path and therefore $\mu$ and $\tau$ will be different in general. Nevertheless, because the second equation in (15) is linear, when considering algorithms based on Newton's method it follows that if the second equation in (15) is satisfied by the starting point, then it will be satisfied by all subsequent points. We will assume that the algorithms under consideration produce points $(x, s, \tau)$ with decreased values of $\tau$ (i.e., $\left.\tau \leq \tau_{0}\right)$ and which belong to a certain neighborhood of the infeasible central path. More precisely we assume that the points belong to a "large" neighborhood of the form

$$
\begin{gathered}
\mathcal{N}_{-\infty}(\nu, \beta):= \\
\left\{(x, s, \tau) \in \mathbb{R}_{++}^{2 n+1} \mid Q x+R s=b-\tau a, \nu \tau e \leq x s, \mu \leq \beta \tau, \tau \leq \tau_{0}\right\},
\end{gathered}
$$

where $0<v<1$ and $\beta>0$ are two given constants. Since in this paper we are only interested in the local convergence, the constants $v$ and $\beta$ may depend on the problem data, including the dimension $n$ of the problem. In [4] we considered a neighborhood depending only on a single parameter

$$
\mathcal{V}_{v}:=\left\{(x, s, \tau) \in \mathbb{R}_{++}^{2 n+1} \mid Q x+R s=b-\tau a, v \tau e \leq x s \leq v^{-1} \tau e, \tau \leq \tau_{0}\right\} .
$$

Since

$$
\nu \tau \leq \mu \leq v^{-1} \tau \text { for all }(x, s, \tau) \in \mathcal{V}_{\nu}
$$

it follows that

$$
\mathcal{V}_{v} \subset \mathcal{N}_{-\infty}\left(v, v^{-1}\right)
$$


and

$$
\mathcal{N}_{-\infty}(\nu, \beta) \subset \mathcal{V}_{\nu} \text { for all } \beta \leq(n v)^{-1}
$$

We denote the feasible set, and the solution set of (10) by

$$
\mathcal{F}:=\left\{(x, s) \in \mathbb{R}_{+}^{n} \times \mathbb{R}_{+}^{n} ; Q x+R s=b\right\},
$$

and

$$
\mathcal{F}^{*}:=\{(x, s) \in \mathcal{F} ; x s=0\} .
$$

From the lemma below it follows that if (10) has a solution then $\mathcal{N}_{-\infty}(\nu, \beta)$ is bounded.

Lemma 1. Assume that $(Q, R) \in P_{*}(\kappa)$ and that $\mathcal{F}^{*}$ is nonempty. Then for any $\left(x^{*}, s^{*}\right) \in \mathcal{F}^{*}$ and $(x, s, \tau) \in \mathcal{N}_{-\infty}(\nu, \beta)$ we have

$$
\begin{gathered}
x^{T} s^{0}+s^{T} x^{0} \leq \beta(1+4 \kappa)\left(2+\zeta^{*}\right) n \tau_{0}, \\
(1-\psi)\left(x^{T} s^{*}+s^{T} x^{*}\right) \leq \beta(1+4 \kappa)\left((1+\psi)+(1-\psi) \zeta^{*}\right) n \tau,
\end{gathered}
$$

where

$$
\psi=\tau / \tau_{0}, \quad \zeta^{*}=\left(\left(x^{0}\right)^{T} s^{*}+\left(s^{0}\right)^{T} x^{*}\right) /\left(\left(x^{0}\right)^{T} s^{0}\right) .
$$

Proof. Since $r=\psi r^{0}$ and $0 \leq \psi \leq 1$ we have

$$
Q\left(\psi x^{0}+(1-\psi) x^{*}-x\right)+R\left(\psi s^{0}+(1-\psi) s^{*}-s\right)=0 .
$$

Using inequalities $\left(x^{*}, s^{*}\right) \geq 0,(x, s)>0$, and (12) we can write

$$
\begin{aligned}
& {\left[\psi x^{0}+(1-\psi) x^{*}-x\right]^{T}\left[\psi s^{0}+(1-\psi) s^{*}-s\right]} \\
& \geq-4 \kappa \sum_{i \in \mathcal{I}_{+}}\left[\psi x^{0}+(1-\psi) x^{*}-x\right]_{i}\left[\psi s^{0}+(1-\psi) s^{*}-s\right]_{i} \\
& \geq-4 \kappa \sum_{i \in \mathcal{I}_{+}}\left(\psi^{2}\left[x^{0}\right]_{i}\left[s^{0}\right]_{i}+(1-\psi) \psi\left(\left[x^{*}\right]_{i}\left[s^{0}\right]_{i}+\left[x^{0}\right]_{i}\left[s^{*}\right]_{i}\right)+[x]_{i}[s]_{i}\right) \\
& \geq-4 \kappa\left(\psi^{2}\left(x^{0}\right)^{T} s^{0}+(1-\psi) \psi\left(\left(x^{*}\right)^{T} s^{0}+\left(x^{0}\right)^{T} s^{*}\right)+x^{T} s\right),
\end{aligned}
$$

where

$$
\mathcal{I}_{+}=\left\{i:\left[\psi x^{0}+(1-\psi) x^{*}-x\right]_{i}\left[\psi s^{0}+(1-\psi) s^{*}-s\right]_{i}>0\right\} .
$$

By expanding (27), we obtain

$$
\begin{aligned}
& {\left[\psi x^{0}+(1-\psi) x^{*}-x\right]^{T}\left[\psi s^{0}+(1-\psi) s^{*}-s\right]} \\
& =\psi \psi^{2} n \mu_{0}+(1-\psi) \psi\left(\left(x^{0}\right)^{T} s^{*}+\left(s^{0}\right)^{T} x^{*}\right) \\
& \quad-\psi\left(\left(x^{0}\right)^{T} s+\left(s^{0}\right)^{T} x\right)+x^{T} s-(1-\psi)\left(s^{T} x^{*}+x^{T} s^{*}\right)+(1-\psi)\left(x^{*}\right)^{T} s^{*},
\end{aligned}
$$

and the desired inequalities (23) and (24) follow by using (28), $\left(x^{*}\right)^{T} s^{*}=0, s^{T} x^{*}+$ $x^{T} s^{*} \geq 0, s^{T} x^{0}+x^{T} s^{0}>0$, and $\mu \leq \beta \tau$. 
In order to study the asymptotic behavior of the points $(x, s, \tau) \in \mathcal{N}_{-\infty}(\nu, \beta)$ as $\tau \rightarrow 0$ we have to examine more carefully the structure of the solution set $\mathcal{F}^{*}$. Let us define three subsets $B, N$ and $J$ of the index set $\{1, \cdots, n\}$ by

$$
\begin{aligned}
B & =\left\{i=1, \cdots, n \mid\left[x^{*}\right]_{i}>0 \text { for at least one }\left(x^{*}, s^{*}\right) \in \mathcal{F}^{*}\right\}, \\
N & =\left\{i=1, \cdots, n \mid\left[s^{*}\right]_{i}>0 \text { for at least one }\left(x^{*}, s^{*}\right) \in \mathcal{F}^{*}\right\}, \\
J & =\left\{i=1, \cdots, n \mid\left[x^{*}\right]_{i}=\left[s^{*}\right]_{i}=0 \text { for all }\left(x^{*}, s^{*}\right) \in \mathcal{F}^{*}\right\} .
\end{aligned}
$$

It is known that the index sets $B, N, J$ form a partition of $\{1, \cdots, n\}$ and that the definition of $B$ and $N$ is independent of the particular solution $\left(x^{*}, s^{*}\right) \in \mathcal{F}^{*}$ (see [6]). In fact it can be shown that

$$
x_{B}^{*}>0, s_{N}^{*}>0, x_{N}^{*}=0, s_{B}^{*}=0
$$

for all $\left(x^{*}, s^{*}\right)$ in the relative interior of $\mathcal{F}^{*}$, and that if $\left(x^{*}, s^{*}\right) \in \mathcal{F}^{*}$ then $x^{*}$ cannot have more than card $(B)$ nonzero entries and $s^{*}$ cannot have more than card $(N)$ nonzero entries. Moreover all the nonzero entries of $x^{*}$ have the corresponding indices in $B$ and all the nonzero entries of $s^{*}$ have the corresponding indices in $N$ (cf. [7], [6]). Therefore any solution satisfying (29) is called a maximal solution. The relative interior of $\mathcal{F}^{*}$ coincides with the set of all maximal solutions. If the set $J$ is empty then a maximal solution is called a strictly complementary solution. We say that the problem is nondegenerate if it has a strictly complementary solution. The set of all strictly complementary solutions of (10) will be denoted by

$$
\mathcal{F}^{c}:=\left\{\left(x^{*}, s^{*}\right) \in \mathcal{F} * ; x^{*}+s^{*}>0\right\} .
$$

Using Lemma 1, the fundamental paper of Monteiro and Wright [15], and the techniques used in the proof of Theorem 1 of Mizuno [13], we obtain the following result.

Proposition 7. If $\mathcal{F}^{*}$ is nonempty, then there exist positive constants $\phi^{\prime}, \phi$ such that

$$
\begin{aligned}
\phi^{\prime} e_{B} \leq x_{B} \leq \phi e_{B}, & \phi^{\prime} \tau e_{N} \leq x_{N} \leq \phi \tau e_{N}, & \phi^{\prime} \sqrt{\tau} e_{J} \leq x_{J} \leq \phi \sqrt{\tau} e_{J}, \\
\phi^{\prime} \tau e_{B} \leq s_{B} \leq \phi \tau e_{B}, & \phi^{\prime} e_{N} \leq s_{N} \leq \phi e_{N}, & \phi^{\prime} \sqrt{\tau} e_{J} \leq s_{J} \leq \phi \sqrt{\tau} e_{J},
\end{aligned}
$$

for all $(x, s, \tau) \in \mathcal{N}_{-\infty}(\nu, \beta)$.

Let us note that since in $\mathcal{N}_{-\infty}(\nu, \beta)$ we have $\mu=\Theta(\tau)$, and therefore we can replace $\tau$ with $\mu$ in the above inequalities. Similar inequalities can be proved for some families of triplets that do not belong to $\mathcal{N}_{-\infty}(\nu, \beta)$. Rather than trying to establish the most general class of such triplets we will assume that the interior-point method to be analyzed produces a sequence of strictly positive vectors $\left(x^{k}, s^{k}\right)$ such that

$$
\begin{aligned}
x_{B}^{k}=\Theta(1), x_{N}^{k} & =\Theta\left(\mu_{k}\right), x_{J}^{k}=\Theta\left(\sqrt{\mu_{k}}\right), \\
s_{B}^{k}=\Theta\left(\mu_{k}\right), s_{N}^{k} & =\Theta(1), s_{J}^{k}=\Theta\left(\sqrt{\mu_{k}}\right), \\
\lim r^{k} & =0 .
\end{aligned}
$$


where, in conformity with (13) and (14) we have denoted

$$
\mu_{k}=\left(\left(x^{k}\right)^{T} s_{k}\right) / n, r^{k}=b-Q x^{k}-R s^{k} .
$$

We are now ready to state the main results of this section, whose proof uses some techniques developed in [26] and [18].

Theorem 1. Suppose that $\mathcal{F}^{*}$ is nonempty and let $\left(x^{k}, s^{k}\right)$ be a sequence positive vectors satisfying (31), such that $\left(\mu_{k}\right)$ converges $R$-linearly to zero. If

$$
\left\|\left(x^{k+1}, s^{k+1}\right)-\left(x^{k}, s^{k}\right)\right\|=O\left(\mu_{k}^{\sigma}\right),
$$

for some constant $\sigma>0$ then the sequence $\left(x^{k}, s^{k}\right)$ converges $R$-linearly to a maximal solution $\left(x^{*}, s^{*}\right)$ of $(10)$. If $\left(\mu_{k}\right)$ converges $R$-linearly with global rate $\rho<1$ then $\left(x^{k}, s^{k}\right)$ converges $R$-linearly with global rate $\rho^{\sigma}$. Moreover if $\left(\mu_{k}\right)$ has any of the properties from Definitions 3, 7, 9, and 11 then so does $\left(x^{k}, s^{k}\right)$.

Proof. If the sequence $\left(\mu_{k}\right)$ converges R-linearly then it converges R-linearly with a certain global rate. Assume that it converges with global R-linear rate $\rho<1$. Then, according to Proposition $6, \mu_{k}^{\sigma}$ converges with global R-linear rate $\eta:=\rho^{\sigma}<1$. Hence there is a constant $C$ such that

$$
\left\|\left(x^{k+1}, s^{k+1}\right)-\left(x^{k}, s^{k}\right)\right\| \leq C \eta^{k}, \forall k \in \mathbb{N} .
$$

It follows that

$$
\left\|\left(x^{k+i}, s^{k+i}\right)-\left(x^{k}, s^{k}\right)\right\| \leq \frac{C}{1-\eta} \eta^{k}, \forall k, i \in \mathbb{N} .
$$

The above relation shows that the sequence $\left(x^{k}, s^{k}\right)$ is Cauchy, so that it converges to some point $\left(x^{*}, s^{*}\right)$. Using (31) we deduce that $\left(x^{*}, s^{*}\right)$ is a maximal solution of (10). Taking $i \rightarrow \infty$ in this relation we obtain

$$
\left\|\left(x^{k}, s^{k}\right)-\left(x^{*}, s^{*}\right)\right\| \leq \frac{C}{1-\eta} \eta^{k}, \forall k \in \mathbb{I N} .
$$

which shows that $\left(x^{k}, s^{k}\right)$ converges R-linearly with global rate $\rho^{\sigma}$.

We note that under the hypothesis of the above theorem the Q-linear convergence of the sequence $\left(\mu_{k}\right)$ does not imply the Q-linear convergence of the sequence $\left(x^{k}, s^{k}\right)$. However from the next theorem it follows that the Q-superlinear convergence of the sequence $\left(\mu_{k}\right)$ implies the Q-superlinear convergence of the sequence $\left(x^{k}, s^{k}\right)$.

Theorem 2. Suppose that $\mathcal{F}^{*}$ is nonempty. Let $\left(x^{k}, s^{k}\right)$ be a sequence of elements of positive vectors satisfying (31) such that $\left(\mu_{k}\right)$ converges $Q$-superlinearly to zero. If

$$
\left\|\left(x^{k+1}, s^{k+1}\right)-\left(x^{k}, s^{k}\right)\right\|=O\left(\mu_{k}^{\sigma}\right),
$$

where $\sigma=.5$ if the HLCP (10) is degenerate and $\sigma=1$ if the HLCP (10) is nondegenerate, then the sequence $\left(x^{k}, s^{k}\right)$ converges $Q$-superlinearly to a maximal solution $\left(x^{*}, s^{*}\right)$ of (10). Moreover if $\left(\mu_{k}\right)$ has any of the properties from Definitions 8, 10 , and 12 then so does $\left(x^{k}, s^{k}\right)$. In particular the $Q$-orders of convergence of $\left(x^{k}, s^{k}\right)$ and $\left(\mu_{k}\right)$ are the same. 
Proof. The convergence of $\left(x^{k}, s^{k}\right)$ towards a maximal solution $\left(x^{*}, s^{*}\right)$ of (10) follows from Theorem 1. If $\left(\mu_{k}\right)$ converges Q-superlinearly to zero then there is a positive integer $K$ such that

$$
\mu_{k+1}^{\sigma} \leq \frac{1}{2} \mu_{k}^{\sigma}, \forall k \geq K
$$

It follows that

$$
\mu_{k+i}^{\sigma} \leq\left(\frac{1}{2}\right)^{i} \mu_{k}^{\sigma}, \forall k \geq K, \forall i \in \mathbb{N}
$$

Proceeding as in the proof of Theorem 1 we deduce that

$$
\left\|\left(x^{k}, s^{k}\right)-\left(x^{*}, s^{*}\right)\right\|=O\left(\mu_{k}^{\sigma}\right) .
$$

(i) If the problem is degenerate, i.e., if $J$ is nonempty, then we have $x_{J}^{*}=0, s_{J}^{*}=0$, and from (31) we obtain

$$
\left\|\left(x^{k}, s^{k}\right)-\left(x^{*}, s^{*}\right)\right\| \geq\left\|\left(x_{J}^{k}, s_{J}^{k}\right)\right\|=\Omega\left(\sqrt{\mu_{k}}\right) .
$$

(ii) If the problem is nondegenerate, i.e., if $J$ is empty, then, by using the fact that $x_{N}^{*}=0, s_{B}^{*}=0$ and (31), we deduce

$$
\left\|\left(x^{k}, s^{k}\right)-\left(x^{*}, s^{*}\right)\right\| \geq \max \left\{\left\|x_{N}^{k}\right\|,\left\|s_{B}^{k}\right\|\right\}=\Omega\left(\mu_{k}\right) .
$$

Hence

$$
\left\|\left(x^{k}, s^{k}\right)-\left(x^{*}, s^{*}\right)\right\|=\Omega\left(\mu_{k}^{\sigma}\right),
$$

where $\sigma=.5$ if the HLCP (10) is degenerate and $\sigma=1$ if the HLCP (10) is nondegenerate. The conclusion of the theorem follows from the above relation, Proposition 4 , and the fact that the Q-superlinear convergence properties of the sequences $\left(\mu_{k}\right)$ and $\left(\mu_{k}^{\sigma}\right)$ are identical.

By using Proposition 5 we can prove in a similar manner the following result.

Theorem 3. Suppose that $\mathcal{F}^{*}$ is nonempty. Let $\left(x^{k}, s^{k}\right)$ be a sequence of elements of positive vectors satisfying (31). Assume there is a constant $\zeta \in(0,1)$ such that

$$
\left\|\left(x^{k+1}, s^{k+1}\right)-\left(x^{k}, s^{k}\right)\right\|=O\left(\mu_{k}^{\sigma \zeta}\right),
$$

where $\sigma=.5$ if the HLCP (10) is degenerate and $\sigma=1$ if the HLCP (10) is nondegenerate. If $\left(\mu_{k}\right)$ converges to zero with strong $Q$-order $\omega$ and $\zeta \omega>1$ then the sequence $\left(x^{k}, s^{k}\right)$ converges to a maximal solution $\left(x^{*}, s^{*}\right)$ of $(10)$ with strong $Q$-order at least $\zeta \omega$. Also, if $\zeta \mathcal{Q}\left(\left(\mu_{k}\right)\right)>1$ then $\mathcal{Q}\left(\left(x^{k}, s^{k}\right)\right) \geq \zeta \mathcal{Q}\left(\left(\mu_{k}\right)\right)$. 


\section{Applications}

We end this paper by applying the results of the previous section to establish the Qsuperlinear convergence of the iteration sequences generated by several well known primal-dual interior point methods. Although all those methods can be generalized for a $P_{*}$-HLCP of the form (10), it is beyond the scope of the present paper to do so. Therefore we will content ourselves to establish the Q-superlinear convergence of the iterates in the framework developed in the original papers.

The simplified MTY algorithm. The simplified MTY method proposed by Gonzaga and Tapia [10] for LP requires only one matrix factorization and two backsolves per iteration while retaining the $O(\sqrt{n} L)$ iteration complexity and the Q-quadratic convergence of the duality gap of the original MTY method. From the analysis in [10] it follows that the iterates generated by the simplified MTY method satisfy $\left\|\left(x^{k}, s^{k}\right)-\left(x^{k+1}, s^{k+1}\right)\right\|=O\left(\mu_{k}\right)$. Since the hypothesis of Theorem 2 are satisfied we deduce that the sequence $\left(x^{k}, s^{k}\right)$ converges Q-quadratically.

The simplified largest step algorithm. The superlinear convergence of the duality gap for the feasible largest step algorithm was first proved by McShane [12] for nondegenerate monotone SLCPs under the assumption that the iteration sequence converges. The results of Bonnans and Gonzaga [2] show that this assumption holds. The largest step method was extended for the infeasible case by Bonnans and Potra [4]. Gonzaga [8] proposed a simplified largest step algorithm and showed that for this algorithm the duality gap converges to zero Q-quadratically. This result was extended to the infeasible case in [4]. From the analysis in the latter papers it follows that the iterates generated by the simplified largest step algorithm satisfy $\left\|\left(x^{k}, s^{k}\right)-\left(x^{k+1}, s^{k+1}\right)\right\|=O\left(\mu_{k}\right)$. Hence the sequence $\left(x^{k}, s^{k}\right)$ converges Q-quadratically.

The LPF+ algorithm of Wright. In [26] Steve Wright proposed an infeasible interiorpoint method for monotone SLCP and proved that if the SLCP is nondegenerate then the duality-gap is Q-subquadratically convergent and the iteration sequence is Rsubquadratically convergent. In his book [27] Wright describes this algorithm for LP and calls it LPF+. Since from [26] we deduce that $\left\|\left(x^{k}, s^{k}\right)-\left(x^{k+1}, s^{k+1}\right)\right\|=O\left(\mu_{k}\right)$ the iteration sequence is not only R-subquadratically convergent, but also Q-subquadratically convergent.

Higher order algorithms. In [3] Bonnans and Gonzaga proposed a high order simplified largest step method that requires asymptotically one matrix factorization and $2 p$ backsolves per iteration. They proved that for this method $\left(\mu_{k}\right)$ converges to zero with strong Q-order at least $p+1$. From Corollary 5.6 of [3] it follows that $\left\|\left(x^{k}, s^{k}\right)-\left(x^{k+1}, s^{k+1}\right)\right\|=O\left(\mu_{k}\right)$. Therefore the sequence $\left(x^{k}, s^{k}\right)$ also converges with strong Q-order at least $p+1$.

Wright and Zhang [28] modified the method in [26] to obtain a method for solving monotone linear complementarity problems that asymptotically requires one matrix factorization and $p$ backsolves at each iteration for which $\mathcal{Q}\left(\left(\mu_{k}\right)\right) \geq p+1$. Potra and Sheng [17] considered a method for solving $P_{*}$ linear complementarity problems with similar convergence properties. From [28] and [17] it can be deduced that $\|\left(x^{k}, s^{k}\right)-$ $\left(x^{k+1}, s^{k+1}\right) \|=O\left(\mu_{k}\right)$ for both method. Hence the iterates produced by both methods are Q-superlinearly convergent and $\mathcal{Q}\left(\left(x^{k}, s^{k}\right)\right) \geq p+1$. 
Stoer, Wechs and Mizuno [22] proposed an infeasible interior-point method for solving (10) that also requires asymptotically one matrix factorization and $p$ backsolves per iteration. The algorithm depends on a constant $\zeta \in(0,1)$. In [22] it is shown that the primal-dual gap converges to zero with strong Q-order at least $p+1-\zeta$ and the iteration sequence converges with the same R-order. It is also shown that the iteration sequence satisfies (31) and $\left\|\left(x^{k}, s^{k}\right)-\left(x^{k+1}, s^{k+1}\right)\right\|=O\left(\mu_{k}^{\zeta}\right)$. From Theorem 3 it follows that if $\zeta(p+1-\zeta)>1$ then the iteration sequence converges with strong Q-order at least $\zeta(p+1-\zeta)$.

Degenerate problems. In all the above results it was assumed that a strictly complementary solution exists. In [19] Potra and Sheng showed that if the problem does not have a strictly complementary solution then the duality gap of their algorithm satisfies $\mathcal{Q}\left(\left(\mu_{k}\right)\right) \geq 1.25$. From their paper it also follows that $\left\|\left(x^{k}, s^{k}\right)-\left(x^{k+1}, s^{k+1}\right)\right\|=$ $O\left(\sqrt{\mu_{k}}\right)$. Taking $\sigma=0.5$ in Theorem 2 we deduce that the iteration sequence is also Q-superlinearly convergent and $\mathcal{Q}\left(\left(x^{k}, s^{k}\right)\right) \geq 1.25$.

Stoer, Wechs and Mizuno [22] showed that in the degenerate case the duality gap of their method converges to zero with strong Q-order at least $(p+1-\zeta) / 2$ and that the iteration sequence converges to a solution $\left(x^{*}, s^{*}\right)$ with the same R-order. They also showed that (31) is satisfied and that $\left\|\left(x^{k}, s^{k}\right)-\left(x^{k+1}, s^{k+1}\right)\right\|=O\left(\mu_{k}^{.5 \zeta}\right)$. If $\zeta$ is chosen such that $\zeta(p+1-\zeta)>2$ then from Theorem 3 it follows that $\left(x^{k}, s^{k}\right)$ converges with strong Q-order at least $\zeta(p+1-\zeta) / 2$. If $\zeta$ is very close to one then the Q-order of covergence of the duality gap and the iteration sequence approach $(p+1) / 2$ from above and below respectively.

Sturm [23] has recently proposed a second order algorithm for degenerate monotone HLCPs for which $\left(\mu_{k}\right)$ converges with strong Q-order at least 1.5. It can be easily shown that the iteration sequence converges with strong R-order at least 1.5. Sturm proves that the sequence of distances between the iterates and the solution set, $\left(\operatorname{dist}\left(\left(x^{k}, s^{k}\right), \mathcal{F}^{*}\right)\right)$, satisfies $\operatorname{dist}\left(\left(x^{k}, s^{k}\right), \mathcal{F}^{*}\right)=O\left(\sqrt{\mu_{k}}\right)$ and claims that the sequence $\left(\operatorname{dist}\left(\left(x^{k}, s^{k}\right), \mathcal{F}^{*}\right)\right)$ is Q-superlinearly convergent. In fact from $\operatorname{dist}\left(\left(x^{k}, s^{k}\right), \mathcal{F}^{*}\right)=O\left(\sqrt{\mu_{k}}\right)$ we can only infer that $\left(\operatorname{dist}\left(\left(x^{k}, s^{k}\right), \mathcal{F}^{*}\right)\right)$ is R-superlinearly convergent. We could prove Qsuperlinear convergence if $\operatorname{dist}\left(\left(x^{k}, s^{k}\right), \mathcal{F}^{*}\right)=\Omega\left(\sqrt{\mu_{k}}\right)$, but it is not very clear if the latter relation holds for the algorithm from [23]. Even if the sequence of the distances to the solution set $\left(\operatorname{dist}\left(\left(x^{k}, s^{k}\right), \mathcal{F}^{*}\right)\right)$ is Q-superlinearly convergent, the problem of the convergence of the iteration sequence remains open. We conjecture that the iteration sequence produced by Sturm's method converges, but only at a linear rate.

Acknowledgements. This paper is dedicated to my friend Alin Popluca who provided a cozy environment and excellent computer facilities in his Arizona home where most of the paper was written. Also, I would like to thank Clovis Gonzaga and two anonymous referees for carefully reading the manuscript, catching several missprints and making valuable suggestions.

\section{References}

1. Anitescu, M., Lesaja, G., Potra, F.A. (1997): An infeasible-interior-point predictor-corrector algorithm for the $P_{*}$-Geometric LCP. Applied Mathematics \& Optimization 36, 203-228

2. Bonnans, J.F., Gonzaga, C.C. (1996): Convergence of interior point algorithms for the monotone linear complementarity problem. Mathematics of Operations Research 21, 1-25 
3. Bonnans, J.F., Gonzaga, C.C. (1997): Fast convergence of the simplified largest step path following algorithm. Mathematical Programming 76, 95-115

4. Bonnans, J.F., Potra, F.A. (1997): On the convergence of the iteration sequence of infeasible path following algorithms for linear complementarity problems. Mathematics of Operations Research 22, 378-407

5. Cottle, R.W., Pang, J.-S., Venkateswaran, V. (1989): Sufficient matrices and the linear complementarity problem. Linear Algebra and Its Applications 114/115, 231-249

6. El-Bakry, A.S., Tapia, R.A., Zhang, Y. (1996): On the convergence rate of Newton interior-point methods in the absence of strict complementarity. Comput. Optim. Appl. 6, 157-167

7. El-Bakry, A.S., Tapia, R.A., Zhang, Y. (1994): A study of indicators for identifying zero variables in interior-point methods. SIAM Review 36, 45-72

8. Gonzaga, C.C. (1997): The largest step path following algorithm for monotone linear complementarity problems. Math. Programming Ser. A 76, 309-332

9. Gonzaga, C.C., Tapia, R.A. (1997): On the convergence of the Mizuno-Todd-Ye algorithm to the analytic center of the solution set. SIAM Journal on Optimization 7, 47-65

10. Gonzaga, C.C., Tapia, R.A. (1997): On the quadratic convergence of the simplified Mizuno-Todd-Ye algorithm for linear programming. SIAM Journal on Optimization 7, 66-85

11. Kojima, M., Mizuno, S., Yoshise, A. (1989): A primal-dual interior point algorithm for linear programming. In: Megiddo, N., ed., Progress in Mathematical Programming, pp. 29-47, Springer, New York

12. McShane, K.A. (1994): Superlinearly convergent $O(\sqrt{n} L)$-iteration interior-point algorithms for linear programming and the monotone linear complementarity problem. SIAM Journal on Optimization 4 247-261

13. Mizuno, S. (1996): A superlinearly convergent infeasible-interior-point algorithm for geometrical LCPs without a strictly complementary condition. Math. Oper. Res. 21, 382-400

14. Mizuno, S., Todd, M.J., Ye, Y. (1993): On adaptive-step primal-dual interior-point algorithms for linear programming. Mathematics of Operations Research 18, 964-981

15. Monteiro, R.D.C., Wright, S.J. (1994): Local convergence of interior-point algorithms for degenerate monotone LCP. Computational Optimization and Applications 3, 131-155

16. Potra, F.A. (1989): On $Q$-order and $R$-order of convergence. Journal of Optimization Theory and Applications 63, 415-431

17. Potra, F.A., Sheng, R. (1997): A large-step infeasible-interior-point method for the $P_{*}$-matrix LCP. SIAM Journal on Optimization 7, 318-335

18. Potra, F.A., Sheng, R. (1998): A path following method for LCP with superlinearly convergent iteration sequence. Ann. Oper. Res. 81, 97-114, Applied mathematical programming and modeling, III (APMOD95) (Uxbridge)

19. Potra, F.A., Sheng, R. (1998): Superlinearly convergent infeasible-interior-point algorithm for degenerate LCP. Journal of Optimization Theory and Applications 97, 249-269

20. Roos, C., Vial, J.-P., Terlaky, T. (1997): Theory and Algorithms for Linear Optimization: An Interior Point Approach. Wiley-Interscience Series in Discrete Mathematics and Optimization. John Wiley and Sons

21. Stoer, J., Wechs, M. (1998): Infeasible-interior-point paths for sufficient linear complementarity problems and their analyticity. Math. Programming Ser. A 83, 407-423

22. Stoer, J., Wechs, M., Mizuno, S. (1998): High order infeasible-interior-point methods for solving sufficient linear complementarity problems. Math. Oper. Res. 23, 832-862

23. Sturm, J.F. (1999): Superlinear convergence of an algorithm for monotone linear complementarity problems, when no strictly complementary solution exists. Mathematics of Operations Research 24, 72-94

24. Tapia, R.A., Zhang, Y., Ye, Y. (1995): On the convergence of the iteration sequence in primal-dual interior-point methods. Mathematical Programming 68, 141-154

25. Väliaho, H. (1996): $P_{*}$-matrices are just sufficient. Linear Algebra and its Applications 239, 103-108

26. Wright, S.J. (1996): A path-following interior-point algorithm for linear and quadratic problems. Annals of Operations Research 62, 103-130

27. Wright, S.J. (1997): Primal-Dual Interior-Point Methods. SIAM Publications, Philadephia

28. Wright, S.J., Zhang, Y. (1996): A superquadratic infeasible-interior-point algorithm for linear complementarity problems. Mathematical Programming 73, 269-289

29. Ye, Y. (1997): Interior Point Algorithms : Theory and Analysis. Wiley-Interscience Series in Discrete Mathematics and Optimization. John Wiley and Sons

30. Ye, Y., Güler, O., Tapia, R.A., Zhang, Y. (1993): A quadratically convergent $O(\sqrt{n} L)$-iteration algorithm for linear programming. Mathematical Programming 59, 151-162 